\title{
Erste Erfahrungen mit dem Blutgruppenanalysator Olympus PK 7100 *
}

L. G. The, J. Beyer

DRK-Blutspendedienst Niedersachsen, Zentralinstitut Springe (Chefarzt: Dr. med. H. Schmitt)

\begin{abstract}
Zusammenfassung:
Der sowohl als autonomer Meßplatz nutzbare, sowie auch in ein übergeordnetes. Host-Computer-System mittels V24-Schnittstelle integrierbare Blutgruppenanalysator Olympus PK 7100 wurde während eines 6monatigen Zeitraumes auf seine Verwendung beim Einsatz von serologischen Routineuntersuchungen im Bereich eines Blutspendedienstes erprobt.
\end{abstract}

Neben einer ausreichend hohen Arbeitsgeschwindigkeit von 240 Proben pro Stunde waren einfache und schnell erlernbare Bedienung des Analysators sowie Zuverlässigkeit seiner Mechanik und Elektronik kennzeichnende Systemvorzüge.

Träger der serologischen Reaktionen ist eine spezielle, wiederverwendungsfähige Mikrotiterplatte, auf der jeweils 10 Probandenproben mit 12 softwaremäßig vordefinierbaren, serologischen Einzelparametern untersucht werden können.

Unter Verwendung dieses Trägers lag die Analysen-Zurückweisungs-Quote ebenso wie der Verbrauch an serologischen Reagenzien an der unteren Grenze vergleichbarer Analysatoren.

Methodische, serologische Verbesserungen sind lediglich im Bereich des Antikörperscreenings zu fordern, da eine Erfassung von klinisch bedeutsamen IgG-Antikörpern zum Zeitpunkt der Erprobung nicht gewährleistet war.

Schlüsselwörter:

Blutgruppenanalysator - Blutgruppenprogramme - Mikrotiterplatte

\section{Summary:}

For six month, the blood grouping analyzer Olympus PK 7100, usable both as an autonomous working place and as a system integrated into a superior host computer by means of a V24 interface, was tested for its practicability in routine serologic tests in a blood transfusion service.

Besides a sufficiently high working speed of 240 samples per hour and operations quick and easy to learn, the reliability of the analyzer's mechanics and electronics were the most impressive characteristics of the system.

The serological reactions take place in a special re-usable microtitre plate. Ten blood donor samples can be tested for 12 programmed single serological parameters each per microtitre plate.

The incidence of erroneous results as well as the consumption rate of serological reagents was within the lower limits of comparable systems.

Only methodical and serological improvements in antibody screening are necessary as clinically relevant IgG antibody detection is not possible at present.

Keywords:

blood grouping analyzer - blood grouping programs - microtitre plate

\section{Einleitung}

Blutgruppenbestimmungen werden bei überregionalen Blutspendediensten derzeitig bevorzugt mit Blutgrupכenanalysatoren durchgeführt, die entweder auf dem Ontinous-flow-Prinzip basieren oder die wiederverwenJungsfähige, großdimensionierte, sektorierte Reaktionsscheiben mit Makroküvetten benutzen (1-4).

Neuerdings wird jedoch auch die Mikrotiterplatte, deren olutgruppenserologische Verwendung erstmals von Negmann und Smithies (5) beschrieben wurde, rationell bei Testdurchführungen von kleinen bis mittelgroßen Analysenserien in der Blutgruppenserologie eingesetzt $(6-9)$.

Für die optimale Bearbeitung großer Analysenserien fehlte es hingegen in diesem Bereich bislang an einem geeigneten System.

Mit dem neuen Blutgruppenanalysator Olympus PK 7100 stellt sich jedoch nunmehr ein System vor, das, auf der

- Hersteller Olympus Optical Co. Ltd., Tokyo 
Basis spezieller, wiederverwendungsfähiger Mikrotiterplatten aufbauend, für die automatische Bestimmung großer Analysenserien eingesetzt werden kann.

Dieser primär auf japanische Blutbankbedürfnisse ausgerichtete Analysator wurde nach entsprechender Adaptation während eines 6 monatigen Zeitraumes auf seine Verwendungsfähigkeit im Routinebetrieb eines überregionalen Blutspendedienstes geprüft.

\section{Material und Methoden}

\subsection{Probenmaterial}

Untersucht wurden in der Regel 1-3 Tage alte, ACDstabilisierte Blutproben mit einer durchschnittlichen Plasmavorverdünnung von etwa 1:3.

Nur bei unzureichender Sedimentation der Erythrozyten wurden die Blutproben zusätzlich zentrifugiert.

\subsection{Testreagentien und serologische Ansätze}

Die für die Bestimmungen im ABO- und Rhesus-Blutgruppensystem verwendeten Testseren und deren Verdünnungsstufen sind der Tab. 1 zu entnehmen.

Für die Serumgegenprobe im ABO-System und die Antikörpersuche wurden eigene Testerythrozyten eingesetzt.

Das Antigenmosaik der Antikörpersuchzellen war dabei speziell auf klinisch bedeutsame Antikörper ausgerichtet.

Die optimale, densitometrisch eingestellte Konzentration der Testerythrozytensuspensionen lag bei 1,8\%.

Die Bestimmung der ABO-Blutgruppen erfolgte im agglutinierenden Ansatz. Die Bestimmung des Rhesus-Faktors und das Antikörperscreening wurden mit enzymbehandelten Erythrozyten durchgeführt $(0,1 \%$ ige bzw. $0,2 \%$ ige gepufferte Bromelin-Lösung, Serva 15250). Inkubationsdauer 60 min bei Raumtemperatur.

\subsection{Olympus-Mikrotiterplatte}

Die wiederverwendungsfähigen Mikrotiterplatten des PK 7100 sind geringfügig größer als die sonst üblichen Einmalplatten (Abmessungen: $92 \mathrm{~mm} \times 130 \mathrm{~mm}$ ).

Pro Mikrotiterplatte können 10 Blutproben mit bis zu 12 Einzelansätzen analysiert werden.

Die Besonderheit der Platte besteht darin, daß die gleichzeitig als Fotometerküvetten genutzten V-förmigen Plattenvertiefungen feinste, konzentrisch angeordnete Stufen aufweisen, die für die Analytik entscheidende Bedeutung besitzen (Abb.1). Ohne Zentrifugation und. Aufschüttlungsvorgänge lagern sich während der Inkubationszeit bei positiven serologischen Reaktionen die agglutinierten Erythrozyten in Form eines mehrschichtigen Zellverbandes über den abgestuften Ringen ein. Optisch resultiert daraus eine gleichmäßige Verteilung der Zellen über den gesamten V-förmigen Boden.

Bei negativem Reaktionsausfall gleiten hingegen die meisten Erythrozyten der Schwerkraft folgend ins Zentrum der V-förmigen Vertiefung, während nur ein geringer Anteil in der Stufenperipherie festgehalten wird.

Aus dem fotometrischen Signalvergleich zwischen Küvettenzentrum und -peripherie ergeben sich die in Abb. 2 aufgeführten Auswertemöglichkeiten.
Tab. 1: Verdünnung und Verbrauch von Antiseren •

\begin{tabular}{llll}
\hline $\begin{array}{l}\text { Anti- } \\
\text { serum }\end{array}$ & Charge & $\begin{array}{l}\text { Ver- } \\
\text { dünnung }\end{array}$ & $\begin{array}{l}\text { Verbrauch/ } \\
1000 \text { Proben }\end{array}$ \\
\hline Anti-A & AA936A & $1: 13$ & $2,0 \mathrm{ml}$ \\
Anti-B & BB426B & $1: 31$ & $0,8 \mathrm{ml}$ \\
Anti-AB & AB321A & $1: 16$ & $1,6 \mathrm{ml}$ \\
Anti-D & DS646B & $1: 18$ & $1,5 \mathrm{ml}$ \\
Anti-CDE & $164 C 1$ & $1: 18$ & $1,5 \mathrm{ml}$ \\
\hline
\end{tabular}

- Ortho Diagnostic Systems Inc. Raritan New Jersey.

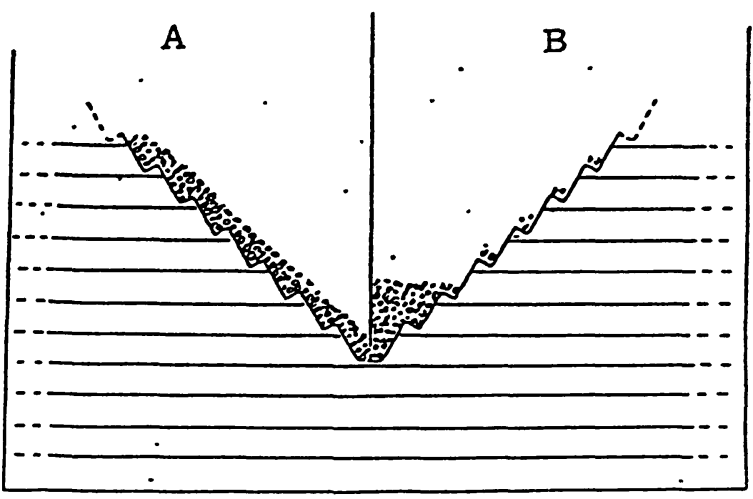

Abb. 1: Vergrößerte schematische Darstellung einer Mikrotiterplattenvertiefung (Seitenansicht) mit schematischem Einlagerungsmuster der Erythrozyten bei positiver (A) und negativer Reaktion (B)

\subsection{Analysenfunktionen des PK 7100}

Zur Erzielung der maximal erreichbaren Analysenfrequenz von 240 Proben/Std. ist der Einsatz der ins Gerät integrierten, positiven Probenidentifikation mit Barcode-Etikettierung der Probenröhrchen erforderlich.

Eine ebenfalls mögliche, serielle Probenbearbeitung mit der Eingabe von maximal achtstelligen Probennummern reduziert die Arbeitsgeschwindigkeit auf etwa 200 Proben/Std.

Zur Analyse werden die Blutproben in Zehner-Racks auf Probencontainer aufgesteckt.

Die simultane Probenentnahme für Plasma und Erythrozyten erfolgt sensorgesteuert über zwei getrennte Entnahmenadeln. Die Proben werden danach zur Vorverdünnung mit $0,9 \%$ iger $\mathrm{NaCl}$ - und Bromelin-Lösung in vom Gerät zyklisch gereinigte Kunststoffbecher einpipettiert. Im Folgeschritt werden die so aufbereiteten Probenbestandteile in die Vertiefungen der automatisch bereitgestellten Mikrotiterplatte unter paralleler Zugabe der serologischen Testreagentien aus .einem Reagenzcontainer transferiert.

Am Ende einer Zehnerserie wird die Mikrotiterplatte einem Inkubator zugeführt.

Nach Ablauf der Inkubationszeit (30 oder $60 \mathrm{~min}$ ) erfolgt die Computerauswertung der fotometrischen Rohdaten mit anschließender Zwischenspeicherung der Endresultate im serologischen Klartext auf einer $8^{\prime \prime}$-Daten-Diskette, wobei zusätzlich eine visuelle Kontrolle der Mikrotiterplatte mit einer optionalen Ergebniskorrektur der Blutgruppenergebnisse vorgesehen ist.

Der gesamte Analysenablauf wird kontinuierlich in Regelkreisen überwacht. Störungen im Ablauf führen zu 
akustischen und optischen Warnmeldungen mit entsprechenden Fehlerangaben. Im Extremfall wird das Analysengerät bis zur Behebung des Fehlers gestoppt.

Zum Datentransfer auf Hostcomputer mit Weiterbearbeitungsmöglichkeit der Analysenergebnisse ist im PK 7100 zusätzlich eine V24-Schnittstelle implementiert.

\section{Ergebnisse}

Die Ergebnisse der Routinebestimmungen im ABO- und Rhesus-Blutgruppensystem sind in Tab. 2 zusammengefaßt.

Fehlbestimmungen ergaben sich in beiden Blutgruppensystemen nicht.

\subsection{ABO-Blutgruppensystem}

Insgesamt wurden in diesem System 20128 Blutproben untersucht. Davon wurden 750 (3,7\%) vom PK 7100 als nicht entscheidbar zurückgewiesen. Dabei lag die Ursache der Zurückweisung nur einmal im Bereich der Antigenbestimmung. In diesem Fall wurde das Merkmal $A_{3}$ bei der Blutgruppe $A_{3} B$ serologisch nicht erfaßt. Die rest!ichen 749 Zurückweisungen ergaben sich im Bereich der Serumgegenprobe des serologischen Gesamtansatzes.
Tab.2: Bestimmungen im ABO- und Rhesus-System

\begin{tabular}{lll}
\hline System & Proben & nicht entschieden \\
\hline ABO & 20128 & $750=3,70 \%$ \\
Rhesus & 20128 & $112=0,55 \%$ \\
\hline
\end{tabular}

Die hauptsächlichen Ursachen der inkompletten Analysen waren

- niedrige Isoagglutinin-Titer, z.T. auch bedingt durch die in der ACD-Blutprobe bereits vorgegebene Plasmavorverdünnung von etwa $1: 3$;

- Verwendung von mehr als vier Tage alten $A_{1}-, A_{2}-$ und B-Testerythrozyten;

- schwach ausgeprägte Blutgruppen der Testerythrozyten, insbesondere bei Blutgruppe $A_{2}$;

- Verwendung der nicht kühlbaren Testerythrozytenansätze über vier Stunden hinaus.

\subsection{Rhesus-Blutgruppensystem}

Bei der Bestimmung des Rhesus-Faktors wurden 112 Proben $(0,55 \%)$ vom Gerät nicht entschieden.
Abb. 2: Fotometrische Ausweriung der Reaktionen in Abhängigiseit von unterschiedlichen Verteiiungsmustem der Erythrozyten $A=$ Ansicht von oben, $B=$ Seirenansicht)

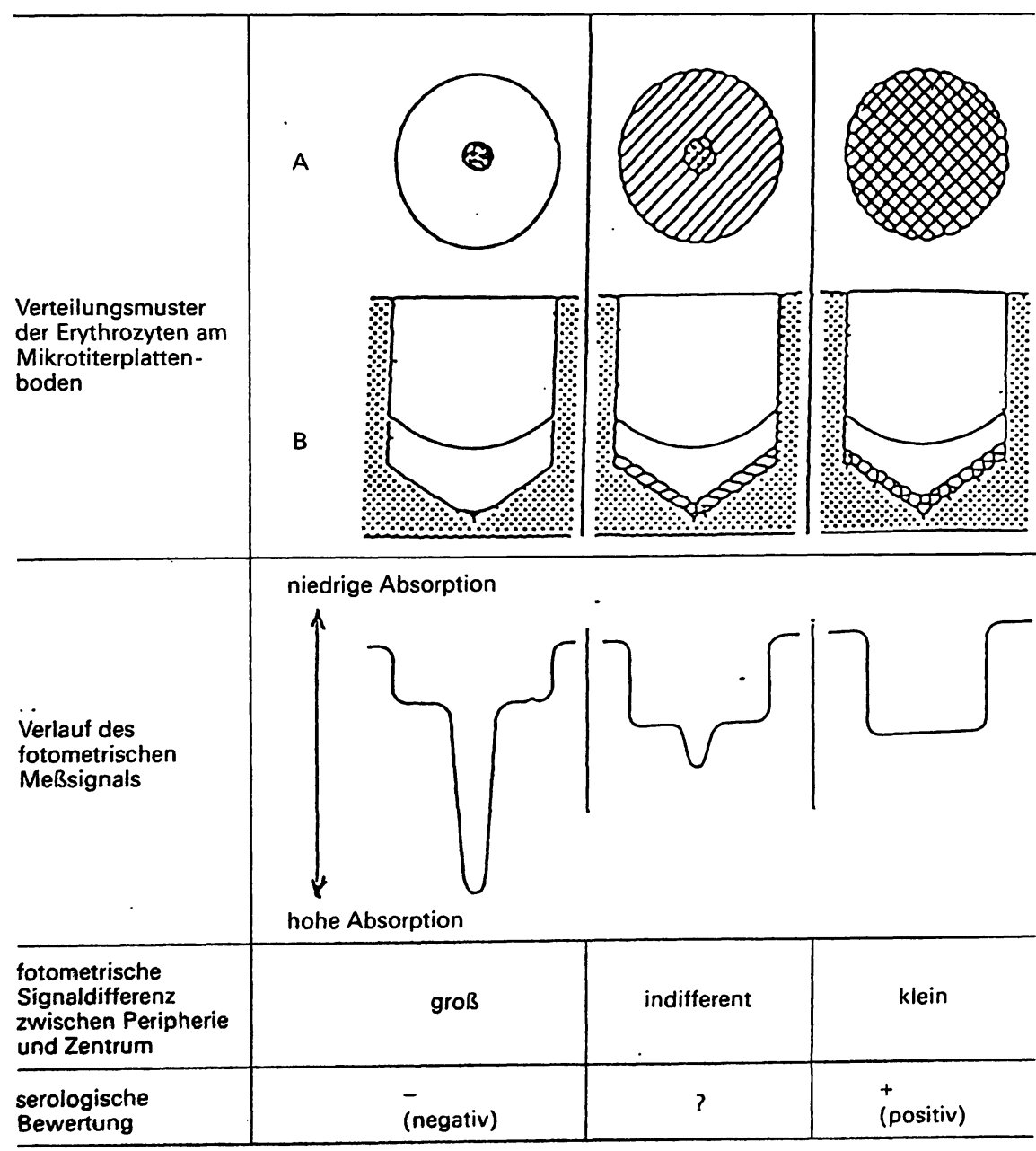


Die Ergebnisverweigerung war dabei weniger serologisch als vielmehr technisch bedingt, indem beim Pipettiervorgang artefiziell vereinzelt kleine Luftbläschen in die $\mathrm{Mi}$ krotiterplattenvertiefungen eingebracht wurden.

Dieses bewirkte eine Störung der fotometrischen Auswertung. Nach Subtraktion dieser Störeffekte ergab sich eine bereinigte serologische Zurückweisungsrate, die unter $0,1 \%$ lag.

\subsection{Antikörpersuchtest}

Da die Durchführung des Antikörpersuchtestes im indirekten Coombstest mit dem Analysator derzeitig nicht möglich ist, wurde nach mehreren Vortestungen ein Antikörperscreening im Enzymansatz durchgeführt. Dabei fand sich methodenbedingt eine relativ hohe Rate positiver Befunde. Von 19876 untersuchten Proben waren 830 $(4,2 \%)$ positiv. In der Vielzahl der positiven Reaktionen konnten bei manueller Gegentestung nur unspezifische Kälteagglutinine nachgewiesen werden. 43 Blutproben $(0,22 \%)$ erbrachten bei der Identifizierung spezifische Antikörper, und zwar 38 Rhesus-, $3 P_{1}$ - und 2 Kell-Antikörper.

\subsection{Weitere Untersuchungen}

Neben den serologischen Routineuntersuchungen erfolgten zusätzlich bei 3000 Blutproben Rhesus-Formelbestimmungen unter Einschluß der Bestimmung des KellMerkmals. Bei einem Antiserumverbrauch zwischen 0,05 und $0,25 \mathrm{ml}$ pro 100 Proben lag die Zurückweisungsrate bei $1 \%$.

Versuche in weiteren Blutgruppensystemen wie im P-, Lewis-, MNS-System Antigenbestimmungen durchzuführen, verliefen ebenfalls erfolgreich. Teilweise mußten hierbei jedoch im Vergleich mit den $A B O$ - und $\mathrm{Rh}-\mathrm{Be}$ stimmungen etwa dreimal höhere Testserummengen eingesetzt werden.

\section{Diskussion}

Im Rahmen der Evaluierung präsentierte sich der Blutgruppenanalysator Olympus PK 7100 als ein kompaktes, gut durchentwickeltes, serologisch fehlerfrei arbeitendes System für die Durchführung von ABO-Blutgruppenund Rhesus-Faktoren-Bestimmungen.

Gravierende technische Pannen wurden während des Erprobungszeitraumes nicht beobachtet.

Der Analysator arbeitet bei mäßiger Geräuschentwicklung präzise und zuverlässig, wobei sich das Gesamtsystem sowohl in serologischer als auch elektronischer Hinsicht durch eine schnell erlernbare, einfache Bedienbarkeit auszeichnet.

Die wesentlichen Schritte des Analysenablaufes werden kontinuierlich von einem ins System integrierten Mikrocomputer überwacht, der auch durch die Ausstattungsmöglichkeit mit verschiedenen Versionen der V24Schnittstelle einen Datentransfer auf Hostcomputer wie beispielsweise auf den Zentralrechner einer Labor-EDV oder zu einem Rechenzentrum unterstützt.

In programmtechnischer Hinsicht erwies sich das Analysensystem zum Zeitpunkt der Erprobung als nur beding flexibel, da trotz der Präsenz von 5 Programmen im Arbeitsspeicher ein Wechsel von einem Routine-Programm zum nächsten, beispielsweise vom ABO-BlutgruppenProgramm zum Rhesusformel-Programm, mit einem 1 stündigen Zeitverlust verbunden war.

Der Zeitverlust resultierte aus dem Umstand, daß die $\mathrm{Be}$ arbeitung der aktuellen Analysen erst komplett abgeschlossen sein mußte, bevor das System mit den neuen Blutproben für das nachfolgende Programm beschickt werden konnte.

Durch überlappend gestaltete Programmabläufe ließe sich dieser Zeitverlust jedoch vermeiden.

Unter Einsatz von serieller oder positiver Probenidentifikation wurde mit dem jeweiligen Routine-Programm ein maximaler Durchsatz von 200 bzw. 240 Proben pro Stunde erreicht, so daß die Gesamtkapazität des Analysators pro achtstündigem Arbeitstag bei einer Stunde Rüstzeit zwischen 1200-1400 Blutgruppenbestimmungen angesetzt werden konnte. Die primäre Analysenzurückweisungsrate lag im üblichen Rahmen anderer Blutgruppenanalysatoren. Durch nachträgliche, visuelle Ablesung von fotometrisch nicht entschiedenen serologischen $\mathrm{Re}$ aktionen mit dem entsprechenden Korrektur-Eintrag auf der Daten-Diskette ließ sich diese Rate sekundär auf etwa $1 \%$ reduzieren.

Im Gegensatz zu den unproblematisch durchführbaren ABO-Blutgruppen- und Rhesus-Faktoren-Bestimmungen war die Durchführung der Antikörpersuche noch nicht optimal gelöst. Speziell die Technik im Enzymansatz erbrachte eine zu hohe Rate von unspezifischen, positiven Befunden und eine um 0,3-0,7\% unter der Norm liegende Rate von spezifischen Antikörpern, wenn man die manuelle Untersuchungstechnik und die kombinierten Techniken des continous-flow-Systems als Vergleichsmethoden heranzieht (10). In diesem Bereich müßten daher weitere serologische Testmodifikationen erarbeitet werden, um eine ausreichend empfindliche und auch eindeutige Erfassung von klinisch bedeutsamen IgG-Antikörperspezifitäten zu erzielen.

Bei zukünftigem Ausbau des Systems wäre es unter anderem zusätzlich wünschenswert, den Analysator auch für die fotometrische Auswertung anderer, auf der Mikrotiterplatte basierender Untersuchungen wie beispielsweise im Bereich der Enzymimmunologie fortzuentwickeln.

Schrifttum:

1. MCNEIL, C., HELMICK, W. M., FERRARI, A.: A preliminary investigation into automatic blood grouping. Vox Sang 8, 235-241 (1963).

2. STURGEON, P. CEDERGREN, B. MCQUISTON, D. Automation of routine blood typing procedures. Vox Sang 8, 438-451 (1963).

3. RECHSTEINER, J., LOCKYER, W. J., FRIEDMAN, L. I.: Overview, history, evaluation and future developments of the AutoGrouper 16-C. Vox Sang 40, 192-200 (1981).

4. MUELLER, A., GARRETTA, M.. HEBERT, M.: Groupamatic System: overview, history of development and evaluation of use. Vox Sang 40, 201-213 (1981).

5. WEGMANN, T. G., SMITHIES, O.: A simple hemagglutination system requiring small amounts of red cells and antibodies. Transfusion 6, 67-73 (1966).

6. CRAWFORD, M. N., GOTTMANN, F. E. GOTTMANN, C. A.: Microplate system for routine use in blood banks laboratories. Transfusion 10, 258-263 (1970).

7. PARKER, J. L., MARCOUX, D. A., HAFLEIGH, E. B., GRUMET, F. C.: Modified microtiter tray method for blood typing. Transfusion 18, 417-422 (1978).

8. LAPINSKI, F. J., CROWLEY, K. M., MERRIT, C. A., HENRY, J. B.: Use of microplate methods in paternity testing. Amer. J. Clin. Path. 70, 766-769 (1978).

9. UTHEMANN, H., ALBERDING, I., GROSSER, M.: Blutgruppenbestimmungen in Mikrotiterplatten. Ärztl. Lab. 30, 259-262 (1984).

10. BEYER, J.: Autoanalyzereinsatz in der Routinediagnostik erythrozytärer Antikörper. Árztl. Lab. 19, 257-266 (1973).

,

Anschrift der Verfasser:

DRK-Blutspendedienst Niedersachsen

Eldagsener Str. 38

3257 Springe 1 


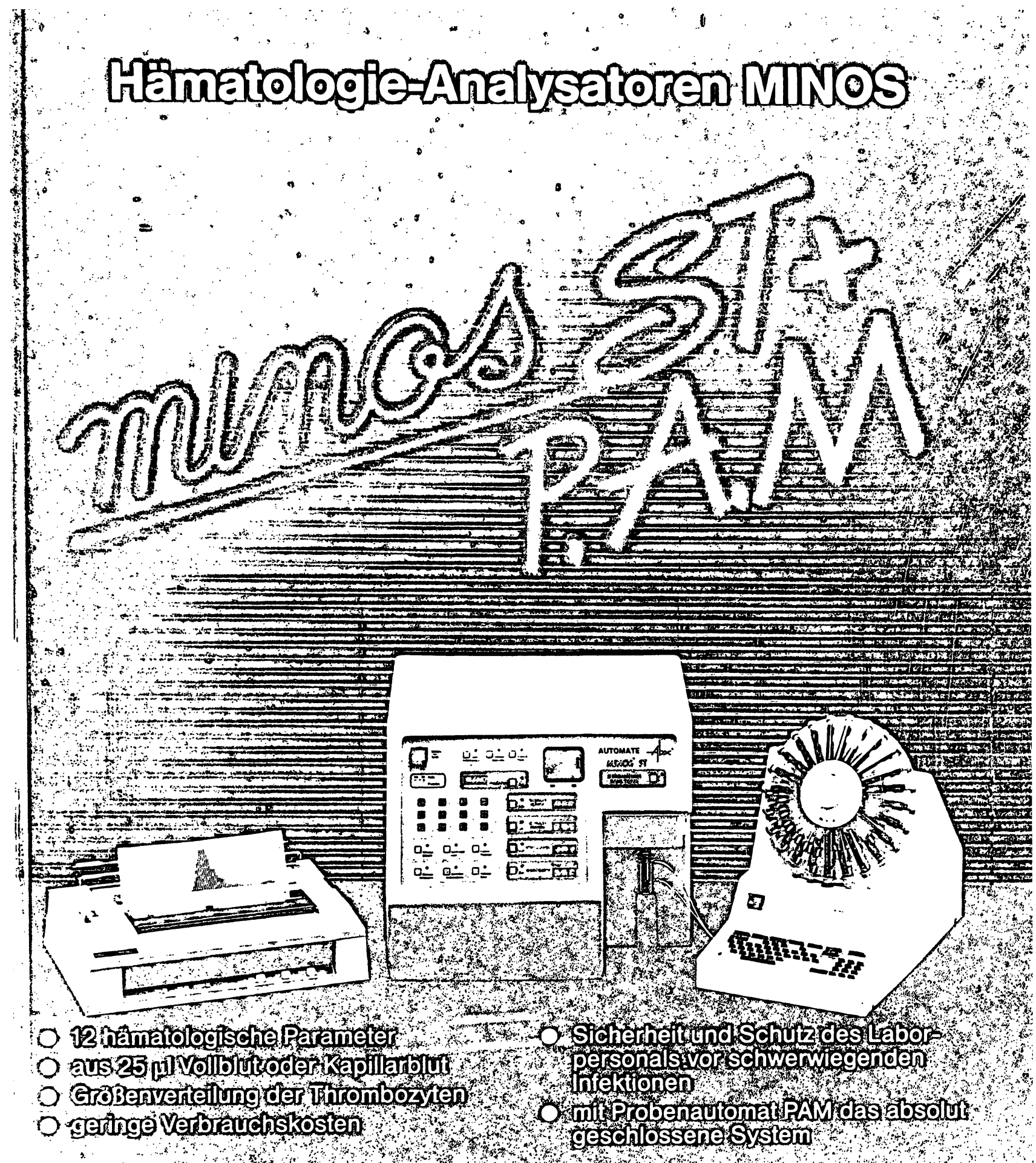

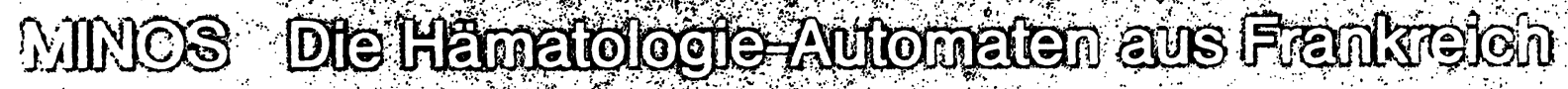

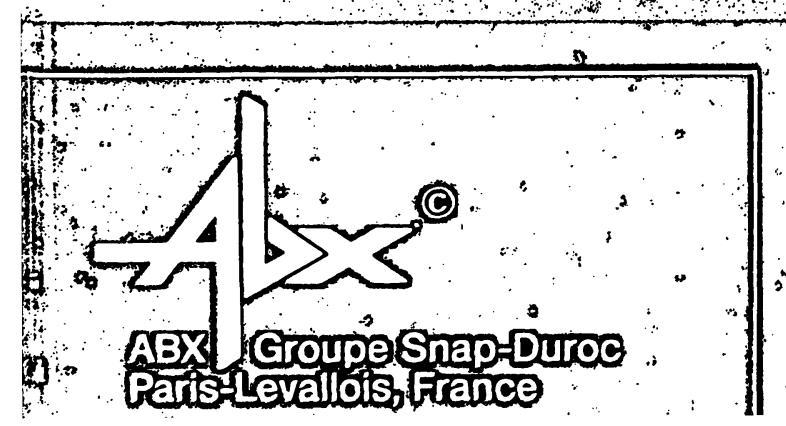

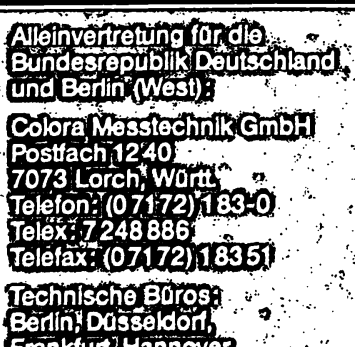

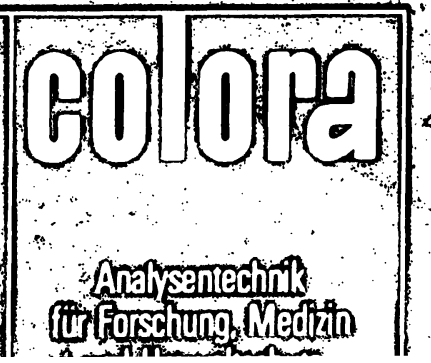




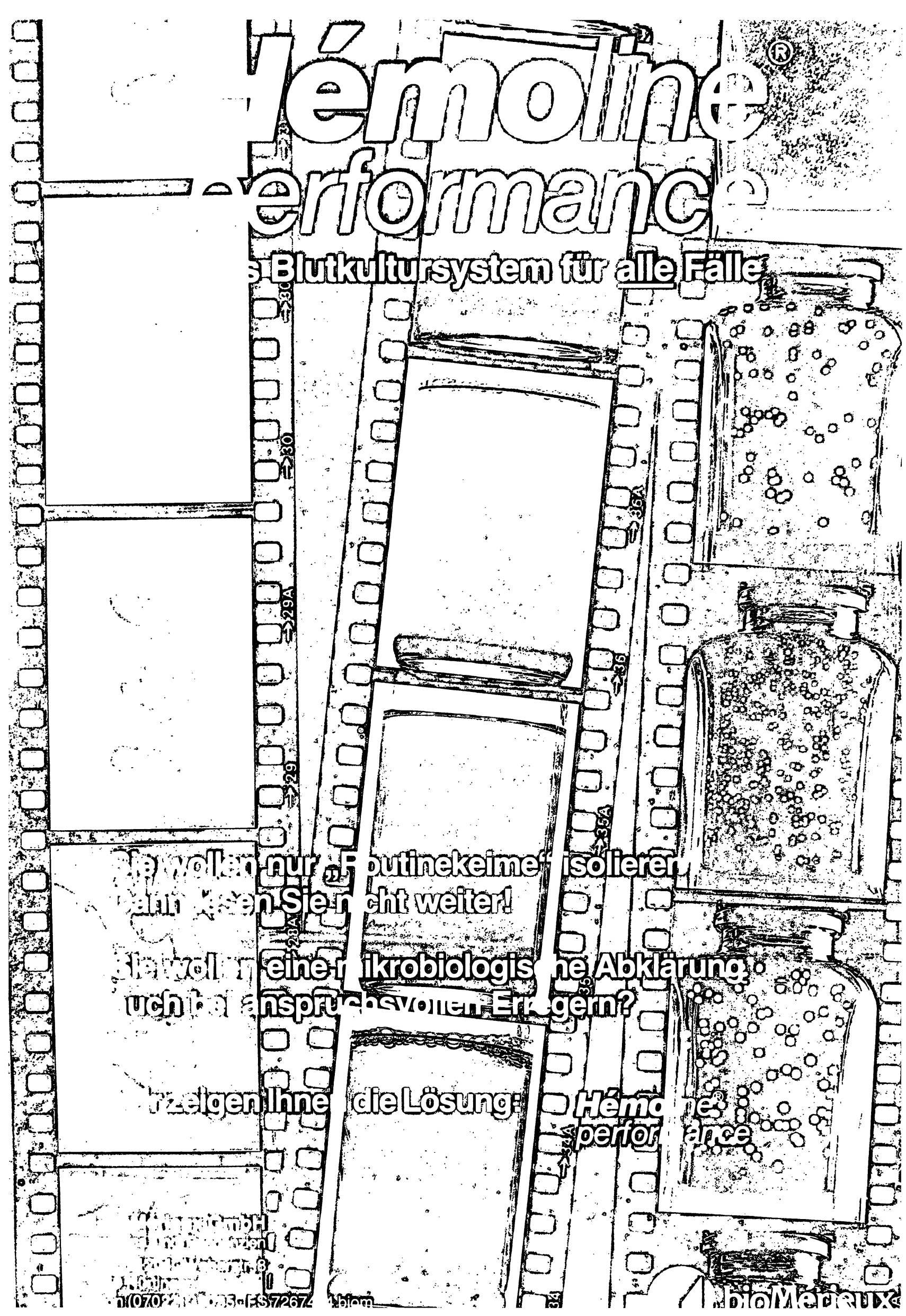

